\title{
Short-term and long-term measures of cortisol in saliva and hair in atypical and non-atypical depression
}

Herane-Vives A, de Angel V, Papadopoulos A, Wise T, Chua K-C, Strawbridge R, Castillo D, Arnone D, Young AH, Cleare AJ. Shortterm and long-term measures of cortisol in saliva and hair in atypical and non-atypical depression.

Background: Atypical depression may show lowered rather than raised short-term cortisol levels. Atypical major depressive episodes (A-MDE) may also be more closely linked to environmental factors and show overlap with somatic symptom disorders. Hair specimens allow measuring long-term cortisol levels.

Methods: Twenty-seven A-MDE and 44 NA-MDE patients and 40 matched controls were tested. Measures of hair cortisol concentration $[\mathrm{HCC}]$ covering the previous 3 months and short-term cortisol parameters (six saliva specimens to assess the cortisol awakening response [CAR] and total daily cortisol output calculated as the area under the curve $[\mathrm{AUCg}]$ ) were taken alongside measures of environmental factors and clinical variables.

Results: There were no differences in HCC between the three groups $(P=0.8)$, and no difference in the CAR $(P=0.95)$. However, A-MDE showed lowered short-term cortisol output (AUCg) compared to controls $(P=0.04)$. A-MDE patients also reported a higher number of daily hassles, and higher levels of fatigue and impaired concentration than NA-MDE.

Conclusions: Normal long-term (HCC) and reduced short-term (AUCg) cortisol levels in A-MDE could suggest a disrupted long-term cortisol rhythm, perhaps affected by environmental factors or by certain symptoms, such as mid-nocturnal insomnia. However, other underlying explanations for these findings should also be investigated in the future.

\author{
A. Herane-Vives ${ }^{1,2}$ iD, \\ V. de Angel ${ }^{1}$, A. Papadopoulos ${ }^{1}$, \\ T. Wise ${ }^{1}$ (D), K.-C. Chua ${ }^{1}$ (iD, \\ R. Strawbridge ${ }^{1}$ (D) D. Castillo ${ }^{3}$, \\ D. Arnone ${ }^{1}$, A. H. Young ${ }^{1}$, \\ A. J. Cleare ${ }^{1}$ iD \\ 1Department of Psychological Medicine, Affective \\ Disorders Research Group, Centre for Affective \\ Disorders, Institute of Psychiatry, Psychology \& \\ Neuroscience, King's College London, London, UK, \\ ${ }^{2}$ Departamento de Clínicas, Facultad de Medicina, \\ Universidad Católica del Norte, Coquimbo, and \\ ${ }^{3}$ Instituto Psiquiátrico José Horwitz Barak, Santiago, \\ Chile
}

Key words: cortisol; affective disorders; classification; depression; diagnosis

Andrés Herane-Vives, Affective Disorders Research Group, Centre for Affective Disorders, Department of Psychological Medicine, Institute of Psychiatry,

Psychology and Neuroscience King's College London, 103 Denmark Hill, London SE5 8AF, UK.

E-mail: andres.herane@kcl.ac.uk

Accepted for publication December 20, 2017

\section{Significant Outcomes}

- Atypical depression differed from non-atypical depression in having reduced cortisol levels using a short-term measure of cortisol - total daily salivary cortisol output - but unchanged levels using a long-term measure - hair cortisol concentration.

- This might suggest that patients with atypical depression show more variable cortisol output, perhaps related to an increased number of daily hassles or to symptoms such as mid-nocturnal insomnia, rather than an overall cortisol concentration alteration.

- The combination of short- and long-term cortisol measures may constitute a more reliable biomarker for delineating the neurobiological features in atypical depression. 


\section{Limitations}

- Salivary cortisol measures were taken on 1 day only, and repeated longitudinal assessments over the period corresponding to the hair cortisol measure would help to confirm whether daily cortisol output is more variable, and more affected by environmental stressors, in atypical depression.

- Cortisol levels in hair are a still relatively novel method of assessment of cortisol, and other confounding variables may have affected the results.

- Patients were free from psychotropic and steroid medication, and other treatments judged likely to affect the HPA axis, but we cannot totally exclude an effect of other prescribed medication.

\section{Introduction}

Increased cortisol levels are frequently found in major depressive disorders $(1,2)$ particularly in patients with melancholic and psychotic features (3). Very little research has, however, been devoted into understanding the biological profile of subtypes of major depression such as atypical depression (4) present in one- to two-thirds of outpatients with major depressive disorders (5). This subtype of an atypical major depressive episode (A-MDE) is characterised by: mood reactivity (a person's mood brightens in response to positive events); significant weight gain or increase in appetite; hypersomnia; leaden paralysis (a heavy, leaden feelings in arms or legs); reversed diurnal mood variation; and a long-standing pattern of interpersonal hypersensitivity (not limited to episodes of mood disturbance) that results in significant social or occupational impairment (DSM-5, (6). Lamers et al. (7) have found that atypical depression may feature decreased, rather than raised cortisol levels when compared to controls using daily salivary measures. Gold and Chrousos have also suggested that there are some specific depressive symptoms, including mood reactivity, profound fatigue and reversed vegetative symptoms, which might specifically be associated with decreased rather than increased short-term cortisol levels (8).

Not all researchers have found clearly decreased levels of cortisol in people with atypical depression. For example, Vreeburg et al. (9) found no significant difference in saliva cortisol reactivity between non-atypical and atypical major depressive episode presentations using the cortisol awakening response (CAR). A review suggested that the association between atypical features and decreased cortisol levels is not well supported by the literature (10) with a particular dearth of evidence comparing cortisol concentrations in atypical depression vs. healthy participants.

Another limitation is the wide range of methods used for measuring cortisol concentrations, particularly in relation to long-term measurements. Hair specimens are a relatively novel source from which to measure chronic levels of cortisol (11) and an improvement upon current methods of assessment (12-15), as this specimen is not affected by the effect of short-term cortisol reactivity or its diurnal variability. Several studies support a positive correlation between the number of acute salivary cortisol measures taken and chronic hair cortisol concentrations (HCC) (16). It has also been started to be used in affective disorders research. Dettenborn et al. (17), for instance, found increased HCC in moderately depressed patients, whilst Wei et al. (18) found that HCC was higher than control subjects for the period during, but not before, a first episode of depression. Hinkelmann et al. (19) assessed hair and saliva specimens in major depression and found raised hair cortisol was confined to those depressed subjects without a history of childhood trauma. Manenschijn et al. (20) found no specific link between HCC and phase of illness in bipolar disorder. More recently, Pochigaeva et al. (21) studied just female depressed patients and found HCC to be lower. As noted earlier, A-MDE might be associated with a different pattern of HPA disturbance. However, to date, no studies have yet investigated HCC in patients with A-MDE, and whether AMDE may be associated with lowered long-term cortisol levels (HCC). Furthermore, no studies have investigated whether a failure to distinguish between A-MDE and non-atypical subtypes (NAMDE) may have led to some of the inconsistent findings in relation to HCC and MDE.

Decreased cortisol levels are also found in somatic symptom disorders such chronic fatigue syndrome (CFS) and fibromyalgia (22-24; Roberts et al., 2004). Indeed, some authors have suggested that atypical depression may be better described as part of this group of disorders $(25,26)$ and that the profound fatigue seen in these states may be the factor most closely linked to reduced HPA activity across the different syndromes (27). The 
association between decreased cortisol levels, somatic symptoms and 'stress-related states' has also led to the suggestion of the collective name of 'stress-related bodily disorders' (28). This term highlights the idea that environmental factors and somatic symptoms may play a substantial role in this subtype of depression in comparison with other subtypes of MDE.

Based on evidence from previous studies indicating (i) lower short-term cortisol secretion in A-MDE, (ii) a significant association between A-MDE and environmental stressors and (ii) the overlap of some symptoms of CFS and symptoms of MDE, we investigated cortisol levels across major depressive episode subtypes and healthy controls. In this study, we focussed on measuring both short- and long-term cortisol levels by collecting saliva and hair specimens, respectively, in NAMDE and A-MDE, but alongside this also assessed the different types of environmental factors that may be a source of stress and the overlapping symptoms within the CFS and MDE case definitions (fatigue and memory impairment). We hypothesised that, compared to NA-MDE, A-MDE would show decreased short- and longterm cortisol levels (7), a greater link with environmental factors (28) and a greater frequency of symptoms that overlap between the MDE and CFS case definitions (25). Furthermore, short- and long-term cortisol level variations in these subtypes of MDE would be also associated with specific clinical variables, such as different symptom patterns and the presence of environmental factors.

\section{Material and methods}

Participants

Participants were recruited in London (UK) and Santiago (Chile) from public advertisements (29) and local psychological therapy services. All patients were assessed by clinical researchers (A.H.V, D.A and T.W. in the UK; V.D. in Chile) who had undergone common training in the methodology and research tools. The four raters showed a high inter-rater agreement on the main diagnostic measures (0.93 kappa coefficient). Forty-four participants with NA-MDE (16 males, mean age $=34.5$ years and mean body mass index $\left.(\mathrm{BMI})=25.4 \mathrm{~kg} / \mathrm{cm}^{2}\right), 27$ with A-MDE (seven males, mean age 31.9 years and mean $\mathrm{BMI}=$ $\left.26.7 \mathrm{~kg} / \mathrm{cm}^{2}\right)$ and 40 controls (11 males, mean age $=33.2$ years and mean $\mathrm{BMI}=24.3 \mathrm{~kg} / \mathrm{cm}^{2}$ ) were recruited so as to match on demographic variables and potential biological confounders (BMI, waist circumference, frequency of hair washing, use of cosmetic treatment, contraceptive pill use, phase of the menstrual cycle, alcohol and tobacco consumption). The controls were predominantly recruited from staff and student volunteers at the Institute of Psychiatry, Psychology and Neurosciences at King's College London and from social media and online classified advertising in the UK and Chile. We selected controls through one-onone matching, by gender and so that the age of the matched control was within 2 years of the patient's age.

Participants were recruited between November 2013 and August 2015. All participants were assessed with psychometric scales validated in both English and Spanish. Diagnostic assessment was completed with the Mini International Neuropsychiatric Interview (MINI; 30). Patients met Axis I DSM-IV criteria for a major depressive episode as part of either a unipolar or bipolar disorder. Healthy controls had no current or past psychiatric diagnoses and reported no history of psychiatric illness in first-degree relatives. Depression severity was established using the Hamilton Depression Rating Scale, 17 item version (HAMD17 and HAMD-21; 31). A severity criterion on the HAMD-17 was not imposed for two reasons; first, as the HAMD-17 emphasises more typical symptoms of depression over atypical symptoms (e.g. it does not score hypersomnia or increased appetite but does score insomnia and reduced appetite), using a severity inclusion would have risked biasing recruitment because A-MDE patients would have been more unwell to meet the same HAMD17 score; and second, so as to reflect the full range of depressive severity seen in out-patients with an MDE. We did, however, require patients to have a score $\geq 11$ to ensure they had clinically significant ongoing depressive symptoms. Depressive symptom ratings using this scale were evaluated on an independent set of patients, and showed high inter-rater reliability across sites (intraclass correlation coefficient $=0.96, P<0.01$ ). Depressive symptoms were also rated using the 16-item Quick Inventory of Depressive Symptoms scale (QIDSC16, 32), which assesses both typical (insomnia, reduced appetite) and atypical (hypersomnia, increased appetite) components of the items with the DSM definition of a Major Depressive Episode. Symptoms of elation were excluded using the Young Mania Rating Scale (YMRS; 33). The allocation of patients to the atypical and non-atypical groups was defined by the Atypical Depression Diagnosis Scale (ADDS; 34). In addition, we totalled two items of the clinician-rated QIDS assessing 'fatigability' and 'impaired concentration' as used in previous studies (35). 


\section{Herane-Vives et al.}

Patients were selected to be free from psychotropic medication or other medication liable to affect the HPA axis for at least 3 months and ongoing psychological interventions at the time of inclusion. All participants provided a hair sample of at least $3 \mathrm{~cm}$ length, and the $3 \mathrm{~cm}$ closest to the scalp was used in the hair cortisol assay. Participants were excluded if they reported any illicit substance use in the previous 3 months or had any unstable medical condition.

For all participants, the frequency and severity of the most common day-to-day environmental disturbances during the month prior to study enrolment were measured using the Hassles Scale (39) and more unexpected environmental factors, such as major life events during the 3 months prior to the study, were assessed using the Recent Life Changes Questionnaire (RLCQ; 37). The occurrence of early life stressors (trauma) was assessed using the Childhood Trauma Questionnaire (CTQ; 38).

The research was approved by the local ethics committee in Chile and in the UK, and written informed consent was obtained from all participants. All participants were compensated for taking part in the research.

\section{Hair samples}

Hair samples were collected from the vertex using standardised procedures, including recording the presence and frequency of confounders potentially affecting hair cortisol concentration. The scalp end was clearly marked, and the $3 \mathrm{~cm}$ of hair nearest the scalp was measured and separated in the laboratory to be used for analysis. Prior to analysis, the hair samples were washed in $1 \mathrm{ml}$ of isopropanol to remove external contaminants, then allowed to dry in a clean air environment for $48 \mathrm{~h}$. Once fully dry, five ceramic balls were added to each tube and the hair samples ground to a powder using am Fast Prep-24 (MP Biomedicals, LLC, Santa Ana, CA, USA). To extract cortisol, $1.75 \mathrm{ml}$ of methanol was added to each sample and the samples incubated for $20 \mathrm{~h}$ whilst rotating the samples constantly.

The hair, methanol and ceramic balls were decanted into a polypropylene tube (Sarstedt AG \& Co, Germany) that separated the ceramic balls from the rest of the mixture. The tube was centrifuged at 3000 RPS to separate the ground hair and methanol, and $1.25 \mathrm{ml}$ of the clear methanol supernatant was decanted into a $2 \mathrm{ml}$ polypropylene cryovial. The methanol was then removed using a vacuum centrifuge (Scan Speed 40; Labgene, Chatel-Saint-Denis, Switzerland) and the tubes frozen at $-80^{\circ} \mathrm{C}$ until required for the cortisol ELISA. Cortisol levels were determined using a commercially available competitive ELISA (Salimetrics LLC, Carlsbad, CA, USA). Samples were thawed and reconstituted with $0.125 \mathrm{ml}$ of Salimetrics cortisol assay diluents, and the samples were then assayed in accordance with the manufacturer's protocol (39). The results were expressed as picograms of cortisol per milligram of hair.

All hair samples were analysed at Salimetrics Laboratory, Cambridge, UK www.salimetrics.c om.

\section{Saliva samples}

Saliva samples were collected a week after the hair samples. All participants were given written information and asked to provide six saliva samples on a single day between Tuesday and Friday using plain salivettes (Sarstedt, Leicester, UK). Participants were instructed not to smoke, eat, drink or brush their teeth for at least an hour before the collection of the samples. The protocol included samples collection (i) immediately after awakening, (ii) 30 min after awakening, (iii) 60 min after awakening, (iv) at noon, (v) at $4 \mathrm{pm}$ and (vi) at $8 \mathrm{pm}$ (24). All participants filled out a questionnaire on sociodemographic details (gender, smoking habits and health problems). Subjects were also instructed to add in the forms whether they experienced any stressors or any other information that they could think would be of relevance and that could be interfering with the study. Moreover, they noted the exact time of sampling for each saliva sample in a patient log. This record was used to assess self-reported compliance. Subjects were then told to put the specimens in the fridge once the collection was finished and send them to the researchers by post within a few days.

Analyses of saliva cortisol concentrations were carried out in the Bethlem Royal Hospital, London, UK, using a previously published method (40). On the arrival to the laboratory, the salivettes were frozen at $-20^{\circ} \mathrm{C}$. After thawing, they were centrifuged at $3500 \mathrm{rev} / \mathrm{min}$ for $10 \mathrm{~min}$, which resulted in a clear supernatant of low viscosity. The saliva specimens were then frozen again in microtubes. Saliva cortisol concentrations were subsequently determined using the 'Immulite' DPC's Immunoassay analyser (www.diagnostics. siemens.com). The plasma cortisol assay of the analyser was suitably modified and then validated for these measurements. A set of 22 cortisol standards in saline were used in each assay to plot a calibration graph. This was highly reproducible with slope of (mean \pm SEM) $0.197 \pm 0.004$. The 
method correlated well with a previously published TR-FIA (40). It had analytical sensitivity of $0.2 \mathrm{nM}$ and inter/intra-assay precision $(\% \mathrm{CV})$ of $<10 \%$ (cortisol concentration range 5-25 nM). All samples from the same subject were analysed in the same run.

The area under the curve with respect to the ground (AUCg) was calculated to estimate the total daily cortisol output based on the collected six samples. Two measures of cortisol reactivity in saliva were also analysed in this study. These included the cortisol awakening response (CAR) and the 30-min delta cortisol value (DELTA). The former was calculated as the area under the curve with respect to increase (AUCi) using the first three-morning saliva samples over a 1 -h period and the latter as the difference between cortisol concentration at the time of waking and the value at 30-min value based on the formula used by Clow et al. (41) and Kunz-Ebrecht et al. (42).

\section{Statistical analyses}

Demographic, clinical and questionnaire data were compared using ANOVA and $t$-tests for continuous variables or chi-square and Fisher's exact test for categorical variables. Differences in cortisol levels among subtypes of depression and healthy controls were tested with ANOVA corrected for multiple comparisons (Bonferroni post hoc test). A linear regression model was used in the A-MDE and NA-MDE groups to assess the relationship between cortisol output and reactivity levels and clinical illness variables, and a logistic regression model for assessing the relationship with categorical dependent illness variables (diurnal mood variation pattern) and number of daily hassles. Two-tailed level for significance was set at $\alpha=0.05$. Eta-squared $\left(\eta^{2}\right)$ was used for calculating different effect sizes (43).

\section{Results}

Forty-four patients with non-atypical and 27 with atypical major depression were matched with 40 healthy controls. Fifty-nine patients were unipolar, and 12 were bipolar. Of the bipolar patients, nine had NA-MDE (comprising $20.5 \%$ of this group), and three had A-MDE (11.1\% of this group). We included depressed participants regardless of a unipolar or bipolar diagnosis, as the focus was on elucidating the influence of atypicality on the biology of major depressive episodes. Unipolar or bipolar depression is a longitudinal definition, whereas this study was focussed on the current episode and atypical features are common in both unipolar and bipolar disorders.
There were no statistically significant differences across the three groups in relation to demographic and clinical variables, such as age, gender, use of contraceptive pills, number of non-psychotropic and non-steroidal medication, number of medical comorbidities and alcohol and tobacco consumption. The two affected groups differed from the healthy controls in relation to clinical parameters, although atypical and non-atypical groups presented with similar depression severity. The atypical group had significantly more somatic symptoms than the non-atypical one, and A-MDE patients were characterised by a higher fatigability and impaired concentration scores on the QIDSC16 $\left(P<0.01, \eta^{2}=0.7\right)$. Healthy participants reported a lower total, and lesser severity, of life events than both depressed groups, although no statistically significant differences were found between the depressed groups. A similar pattern was observed in relation to the occurrence of 'hassles' in comparison with healthy participants, with the noticeable difference that these environmental factors were more common $\left(P<0.01, \eta^{2}=0.4\right)$, but not more severe in the atypical vs. the non-atypical group (see Table 1 for details). Biological parameters that could affect cortisol concentrations such as BMI, waist circumference and menstrual cycle phase did not differ between groups (see Table 2).

\section{Hair cortisol concentrations}

Hair cortisol concentrations did not differ between groups; values were respectively 8.3 (SD: 3.9) pg/ $\mathrm{mg}$ in the healthy participants, 8.7 (SD: 4.5) $\mathrm{pg} / \mathrm{mg}$ in the non-atypical group and 7.8 (SD: 4.8) $\mathrm{pg} / \mathrm{mg}$ in the atypical group $(F(2,108)=0.31, P=0.8$, $\left.\eta^{2}<0.05\right)$.

\section{Saliva cortisol concentrations}

Atypical depression was characterised by significantly lower total daily cortisol output compared with healthy participants (Fig. 1); AUCg values were 94.5 (SD: 30.1) nmol/l.h vs. 120.8 (SD: 39.0) nmol/l.h, respectively $(F(2,82)=3.53, P=0.04$, $\eta^{2}=0.08$; Table 3$)$. There was no statistical difference between either of these groups and the nonatypical group (113.8 (SD: 36.1) nmol/1.h). The CAR measured as AUCi did not differ across the three groups; values were 1.2 (SD: 7.3) $\mathrm{nmol} / \mathrm{l} \cdot \mathrm{h}$ in healthy controls, $1.6(\mathrm{SD}: 5.3) \mathrm{nmol} / \mathrm{l} \cdot \mathrm{h}$ in the atypical group and 1.2 (SD: 4.9) $\mathrm{nmol} / \mathrm{l} \cdot \mathrm{h}$ in the typical group, $\left(F(2,84)=0.04, P=0.95, \eta^{2}<0.01\right)$. Similarly, the DELTA did not differ statistically between groups; values were 2.3 (SD: 6.7) nmol/l.h 


\section{Herane-Vives et al.}

Table 1. Clinical and demographic characteristics

\begin{tabular}{|c|c|c|c|c|c|}
\hline & Healthy controls & Non-atypical group & Atypical group & $P$ value ${ }^{*}$ & Post hoc test \\
\hline N. Total & 40 & 44 & 27 & NA & \\
\hline Mean Age in years (SD) & $33.2(8.9)$ & $34.5(11.7)$ & $31.9(8.3)$ & 0.55 & \\
\hline$M / F$ & $11 / 29$ & $16 / 28$ & $7 / 20$ & 0.56 & \\
\hline N. Employed (n) (\%) & $38(95.0)$ & $35(79.6)$ & $22(81.9)$ & 0.1 & \\
\hline N. Episodes; Mean (SD) & $0(0)$ & $3.9(9.7)$ & $3(4.1)$ & 0.02 & $A, B$ \\
\hline N. Admissions; Mean (SD) & $0(0)$ & $0.2(0.4)$ & $0.2(0.7)$ & 0.12 & $A, B$ \\
\hline Duration of IIIness (weeks);Mean (SD) & $0(0)$ & $85.9(119.75)$ & $97.3(156.3)$ & $<0.01$ & $A, B$ \\
\hline HAMD-17; Mean (SD) & $0.3(0.9)$ & $17.7(5.8)$ & $16.7(4.4)$ & $<0.01$ & $A, B$ \\
\hline QIDS; Mean (SD) & $0.5(1.4)$ & $17.2(4.8)$ & $18.6(4.7)$ & $<0.01$ & $A, B$ \\
\hline YMRS; Mean (SD) & $0.1(0.3)$ & $1.1(1.7)$ & $1.2(1.6)$ & $<0.01$ & $A, B$ \\
\hline Concentration \& fatigue factor; mean (SD) & $0.1(0.3)$ & $3.2(1.5)$ & $3.9(0.9)$ & $<0.01$ & $A, B, C$ \\
\hline QIDS-C items; Mean (SD) & $0.5(1.4)$ & $17.2(4.8)$ & $18.6(4.7)$ & $<0.01$ & $A, B$ \\
\hline Early life trauma(CTO), n (SD) & $8(20.0)$ & $22(50.0)$ & $17(63.0)$ & $<0.01$ & $A, B$ \\
\hline Life events $\leq 3$ months, mean (SD) & $100.7(162.1)$ & 308.2 (260.9) & $337.6(345.6)$ & 0.003 & $A, B$ \\
\hline Severe life events $\leq 3$ months, mean (SD) & $6.0(15)$ & $30.0(68.2)$ & $17.0(63.0)$ & $<0.01$ & $A, B$ \\
\hline Mean 'Hassles' $\leq 1$ month, mean (SD) & $12.1(9.1)$ & $38.1(17.5)$ & $51.5(28.8)$ & $<0.01$ & $A, B, C$ \\
\hline N. Severe 'Hassles' $\leq 1$ month (\%) & $1.0(2.5)$ & $21.0(47.7)$ & $14.0(51.9)$ & $<0.01$ & $A, B$ \\
\hline \multicolumn{6}{|l|}{ N. of participants in each ADDS category $(\%) \dagger$} \\
\hline Non-reactive depression & $0(0)$ & $32(72.7)$ & $0(0)$ & & \\
\hline Simple reactive depression & $0(0)$ & $12(27.2)$ & $0(0)$ & & \\
\hline Probable atypical depression & $0(0)$ & $0(0)$ & $14(51.9)$ & & \\
\hline Definitive atypical depression & $0(0)$ & $0(0)$ & $13(48.1)$ & & \\
\hline
\end{tabular}

A: Controls different from NA-MDE; B: Controls different from A-MDE; C: A-MDE different from NA-MDE $\infty$ : no differences according Bonferroni post hoc test; M: Males; F: Females; SD: Standard deviation; CTQ: Childhood Trauma Questionnaire; HAMD-17: Hamilton Depression Rating scale, 17 items; OIDS: Quick Inventory of Depressive Symptoms scale; OIDS-C: Clinician-rated QIDS; YMRS: Young Mania Rating Scale; ADDS: Atypical Depression Diagnosis Scale.

${ }^{*} P$-value significant at 0.05 level using either Anova or chi-squared test.

$\dagger$ The allocation of depressed participants to either the non-atypical or the atypical group was determined by the ADDS. The NA-MDE group comprised the ADDS categories: (i) 'non-reactive depression' and (ii) 'simple reactive depression', and the A-MDE group comprised the ADDS categories (iii) 'probable atypical depression' and (iv) 'a definite atypical depression'.

Table 2. Distribution of biological variables in the participant groups

\begin{tabular}{lcccc}
\hline & $\begin{array}{c}\text { Healthy } \\
\text { controls }\end{array}$ & $\begin{array}{c}\text { Non-atypical } \\
\text { group }\end{array}$ & $\begin{array}{c}\text { Atypical } \\
\text { group }\end{array}$ & $P$ value \\
\hline N. Total & 40 & 44 & 27 & \\
N. Tobacco users (\%) & $6(15.0)$ & $9(20.5)$ & $7(25.9)$ & 0.57 \\
N. Alcohol users (\%) & $31(77.5)$ & $33(75.0)$ & $20(74.0)$ & 0.84 \\
Mean BMl in kg/m ${ }^{2}$ (SD) & $24.3(3.6)$ & $25.4(5.2)$ & $26.7(4.7)$ & 0.11 \\
Mean waist circumference & $82.1(10.7)$ & $86.1(14.0)$ & $88.8(13.0)$ & 0.1 \\
in cm (SD) & & & & \\
N.Non-psychotropic & $19(47.5)$ & $18(40.9)$ & $11(40.7)$ & 0.52 \\
$\quad$ Medication (\%) & & & & \\
N. Medical comorbidities (\%) & $2(5.0)$ & $8(18.1)$ & $5(18.5)$ & 0.2 \\
Mean hair washes/week (SD) & $4.5(1.7)$ & $4.4(2.1)$ & $3.9(2.0)$ & 0.54 \\
Cosmetic treatments* (\%) & $12(30.0)$ & $14(31.8)$ & $7(25.9)$ & 0.9 \\
N. Females only & 29 & 28 & 20 & \\
$\quad$ N. Oral Contraceptive use (\%) & $10(34.4)$ & $4(14.3)$ & $1(5.0)$ & 0.06 \\
$\quad$ N. Follicular phase (\%) & $9(31.0)$ & $6(21.4)$ & $7(35.0)$ & 0.39 \\
\hline
\end{tabular}

*Dyeing, bleaching, permanent straightening or waving; BMI: Body Mass Index.

in healthy controls, 2.7 (SD: 7.8) nmol/1.h in the atypical group and 3.1 (SD: 6.4) nmol/l.h in the typical group, $\quad(F(2,85)=0.12, \quad P=0.88$, $\left.\eta^{2}<0.01\right)$.

Regression model results

In relation to the different types of sleep disturbance, in A-MDE, mid-nocturnal insomnia was

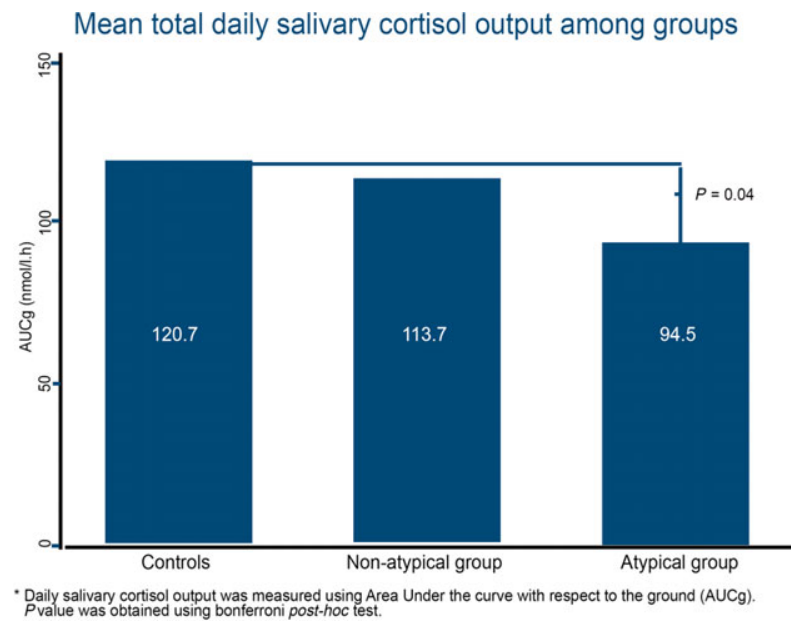

Fig. 1. Mean total daily salivary cortisol output among groups. *Daily salivary cortisol output was measured using area under the curve with respect to the ground (AUCg). $P$ value was obtained using Bonferroni post hoc test.

significantly associated with decreased short-term daily salivary cortisol output (AUCg) $(\beta=-9.2$, $P=0.04, \quad$ CI95\% $=-18.3, \quad-0.1) \quad$ and with decreased cortisol reactivity measures, including the CAR $(\beta=-1.7, P=0.01, \quad$ CI95\% $=-3.2$, $-0.4)$ and DELTA $(\beta=-2.3, \quad P=0.02$, 
Table 3. Cortisol levels in A-MDE, NA-MDE and controls using hair and saliva measures

\begin{tabular}{|c|c|c|c|c|c|c|c|c|c|c|c|c|c|}
\hline \multirow{2}{*}{$\begin{array}{l}\text { Group } \\
\text { Measure }\end{array}$} & \multicolumn{4}{|c|}{ A-MDE } & \multicolumn{4}{|c|}{ NA-MDE } & \multicolumn{4}{|c|}{ Controls } & \multirow[b]{2}{*}{$P$-value } \\
\hline & 01 & Median & Mean (SD) & 03 & 01 & Median & Mean (SD) & 03 & 01 & Median & Mean (SD) & 03 & \\
\hline $\mathrm{HCC}(\mathrm{pg} / \mathrm{mg})$ & 4.1 & 7.8 & $7.8(4.8)$ & 9.6 & 5.5 & 7.6 & $8.7(4.5)$ & 11.0 & 5.5 & 7.1 & $8.3(3.9)$ & 9.8 & 0.80 \\
\hline CAR (nmol/l-h) & -1.2 & -0.4 & $1.2(4.9)$ & 3.8 & -3.5 & 1.7 & $1.6(5.3)$ & 6.0 & -1.3 & -0.4 & $1.2(7.3)$ & 3.8 & 0.95 \\
\hline DELTA cortisol (nmol/l-h) & -0.8 & 1.0 & $3.1(6.4)$ & 6.7 & -2.7 & 2.6 & $2.7(7.8)$ & 10.2 & -1.1 & 3.0 & $2.3(6.7)$ & 6.0 & 0.88 \\
\hline AUCg (nmol//.h) & 74.1 & 91.3 & $94.5(30.1)$ & 117.1 & 88.0 & 110.3 & $113.8(36.1)$ & 136.9 & 97.3 & 112.6 & $1208(39.0)$ & 140.5 & 0.04 \\
\hline
\end{tabular}

$P$-values were calculated using ANOVA.

HCC, hair cortisol concentration; CAR, Cortisol awakening response over 60 min measured as area under the curve with respect to increase (AUCi); DELTA-Cortisol, increase in cortisol over baseline at 30 min after awakening; $\mathrm{AUCg}$, total daily cortisol output measured as area under the curve with respect to ground.

CI95\% $=-4 .,-0.4)$. On the other hand, in NAMDE, hypersomnia was associated with decreased AUCg $\quad(\beta=-15.9, \quad P=0.02, \quad$ CI95\% $=-29.5$, $-2.3)$ and $\operatorname{CAR}(\beta=-2.5, P=0.01, \mathrm{CI} 95 \%$ $=-4.5,-0.6)$ (Table 4).

Fatigue showed opposite tendencies in relation to its association with short- and long-term cortisol levels in A-MDE. Thus, while decreased energy levels showed a trend to being associated with increased short-term daily salivary cortisol output (AUCg) $\quad(\beta=21.6, \quad P=0.07, \quad$ CI $95 \%=-2.5$, $45.9)$, the same variable tended to be associated with decreased long-term cortisol levels (HCC) $(\beta=-3.0, P=0.11$, CI95\% $=-6.9,0.8)$. Conversely, fatigue was significantly associated with increased DELTA in NA-MDE $(\beta=4.3$, $P=0.03$, CI95\% $=0.3,8.3$ ).

The other MDE/CFS overlapping symptom (memory impairment) also tended to be associated with cortisol level alterations, with a trend level association with increased $\mathrm{HCC} \quad(\beta=2.4$, $P=0.08$, CI95\% $=-0.3,5.2)$ in A-MDE. Other non-overlapping MDE symptoms, such as weight decrease $(\beta=-1.1, P=0.05, \mathrm{CI} 95 \%=-2.1,0.0)$ and evening-worsening mood $(\beta=-3.7, P=0.03$, $\mathrm{CI} 95 \%=-7.1,-0.3)$ were significantly associated with decreased HCC in NA-MDE.

Daily hassles were also associated with cortisol concentration variations in MDE. However, this time, an increased number of daily hassles was associated with decreased cortisol reactivity (DELTA) $(\beta=-0.2, \quad P=0.05, \quad$ CI95\% $=-0.3$, $0.0)$ in NA-MDE (Table 4). The association between daily hassles and some of those overlapping and non-overlapping symptoms which were, in turn, related to cortisol concentration alterations in our sample were only apparent in AMDE. For instance, increased daily hassles were associated with increased fatigue $(\beta<0.1$, $P=0.04$, CI95\% $=0.0,0.0)$ in A-MDE, but not in NA-MDE $(\beta<-0.1, P=0.14$, CI95\% $=-0.1$, $0.0)$. The same situation was observed in relation to mid-nocturnal insomnia in which increases in daily hassles tended again to be associated with this type of sleep disturbance in A-MDE $(\beta<0.1$, $P=0.09$, CI95\% $=0.0,0.0)$, but not in NA-MDE $(\beta<0.1, \quad P=0.86, \quad$ CI95\% $=-0.1,0.0)$. Conversely, increases in the number of these environmental factors were not related with worsened hypersomnia, either in A-MDE $(\beta<-0.1$, $P=0.70$, CI95\% $=-0.1,0.1)$ or in NA-MDE $(\beta<-0.1, \quad P=0.33, \mathrm{CI} 95 \%=-0.1,0.0)$. The same lack of association was found between daily hassles and impaired concentration in A-MDE $(\beta<0.1, P=0.68$, CI95\% $=-0.1,0.1)$ and in NA-MDE $(\beta<-0.1, P=0.15, \quad$ CI95\% $=-0.1$, 0.0 in NA-MDE), with evening-worsening mood in A-MDE $(\beta<0.1, P=0.17$, CI95\% $=-0.1$, $0.1)$ and in NA-MDE $(\beta<-0.1, \quad P=0.48$, CI95\% $=-0.1,0.0)$ and with decreased weight in A-MDE $(\beta<-0.1, P=0.36$, CI95\% $=-0.1,0.0)$ and in NA-MDE $(\beta<-0.1, P=0.48, \mathrm{CI} 95 \%$ $=-0.1,0.1)$.

\section{Discussion}

We did not find long-term cortisol secretion alteration using hair specimens, nor differences in short-term cortisol reactivity using those salivary measures designed for measuring that cortisol parameter, such as CAR and DELTA, in either AMDE or NA-MDE. However, we did find decreased short-term cortisol secretion using a salivary measure of total cortisol daily output (AUCg) in A-MDE. A-MDE patients also reported that they had been exposed to an increased number of those environmental factors that are commonly faced during daily activities, known as daily hassles, in comparison with NA-MDE. However, life events, the other types of environmental factor, which are usually more infrequent and unexpected in their appearance, did not differ between these depressive groups. Finally, those somatic symptoms that overlap between MDE and CFS definitions, such as memory impairment and fatigue, were also more marked in A-MDE in comparison with NA-MDE. Although there were trends for these overlapping symptoms to be associated with 
Herane-Vives et al.

Table 4. Regression analyses of relationships between different clinical variables and cortisol levels by group

\begin{tabular}{|c|c|c|c|c|c|c|c|c|c|c|}
\hline \multirow{2}{*}{\multicolumn{2}{|c|}{ Variables }} & \multicolumn{9}{|c|}{ Groups } \\
\hline & & \multicolumn{3}{|c|}{ Controls } & \multicolumn{3}{|c|}{ Non-atypical MDE } & \multicolumn{3}{|c|}{ Atypical MDE } \\
\hline Clinical variables $\dagger$ & Cortisol measure: & $\beta$ & $P$-value & Cl95\% & $\beta$ & $P$-value & $\mathrm{Cl} 95 \%$ & $\beta$ & $P$-value & $\mathrm{Cl} 95 \%$ \\
\hline \multirow[t]{4}{*}{ Sleep onset insomnia } & $\mathrm{HCC}$ & -4.2 & 0.28 & $-12.2,3.7$ & -0.1 & 0.80 & $-1.2,0.9$ & -0.1 & 0.95 & $-1.5,1.4$ \\
\hline & AUCg & 28.4 & 0.48 & $-52.6,109.5$ & -3.4 & 0.50 & $-14.0,7.0$ & 4.0 & 0.42 & $-6.3,14.3$ \\
\hline & CAR & 0.4 & 0.96 & $-14.9,15.7$ & -0.7 & 0.33 & $-2.3,6.1$ & 0.6 & 0.42 & $-1.0,2.3$ \\
\hline & Delta & 0.1 & 0.99 & $-14.1,14.3$ & -0.3 & 0.75 & $-2.6,1.9$ & 0.6 & 0.52 & $-1.5,7.0$ \\
\hline \multirow[t]{4}{*}{ Mid-nocturnal insomnia } & $\mathrm{HCC}$ & 0.6 & 0.66 & $-2.1,3.2$ & -0.8 & 0.14 & $-1.8,0.3$ & 1.0 & 0.12 & $-0.3,2.4$ \\
\hline & AUCg & $0.0 \S$ & - & - & -1.7 & 0.74 & $-12.6,9.2$ & -9.2 & $0.04^{*}$ & $-18.3,-0.1$ \\
\hline & CAR & $0.0 \S$ & - & - & 0.2 & 0.78 & $-1.4,1.8$ & -1.7 & $0.01^{*}$ & $-3.2,-0.4$ \\
\hline & Delta & $0.0 \S$ & - & - & 0.8 & 0.48 & $-1.5,3.1$ & -2.3 & $0.02^{*}$ & $-4.1,-0.4$ \\
\hline \multirow[t]{4}{*}{ Early-morning insomnia } & $\mathrm{HCC}$ & 1.7 & 0.66 & $-6.3,9.8$ & $<0.1$ & 0.94 & $-1.2,1.3$ & 0.5 & 0.62 & $-1.5-2.5$ \\
\hline & AUCg & $0.0 \S$ & - & - & 12.0 & 0.07 & $-1.2,25.2$ & 6.3 & 0.35 & $-7.5,20.1$ \\
\hline & CAR & $0.0 \S$ & - & - & 0.5 & 0.64 & $-1.6,2.5$ & -1.2 & 0.26 & $-3.4,0.9$ \\
\hline & Delta & $0.0 \S$ & - & - & 1.0 & 0.50 & $-2.0,4.0$ & -2.4 & 0.08 & $-5.2,0.3$ \\
\hline \multirow[t]{4}{*}{ Hypersomnia } & $\mathrm{HCC}$ & 1.2 & 0.75 & $-6.8,9.3$ & 1.0 & 0.17 & $-0.5,2.5$ & -1.2 & 0.24 & $-3.2,0.8$ \\
\hline & AUCg & -34.4 & 0.39 & $-115.1,46.3$ & -15.9 & $0.02^{*}$ & $-29.5,-2.3$ & 8.8 & 0.21 & $-5.7,23.3$ \\
\hline & CAR & 6.5 & 0.38 & $-8.6,21.7$ & -2.5 & $0.01^{*}$ & $-4.5,-0.6$ & 0.1 & 0.94 & $-2.4,2.5$ \\
\hline & Delta & 6.6 & 0.34 & $-7.4,20.5$ & -3.3 & 0.06 & $-6.8,0.1$ & -0.5 & 0.76 & $-3.6,2.7$ \\
\hline \multirow[t]{4}{*}{ Appetite decreased } & $\mathrm{HCC}$ & 2.6 & 0.36 & $-3.1,8.3$ & -1.3 & 0.08 & $-2.7,0.2$ & -0.5 & 0.65 & $-2.7,1.7$ \\
\hline & AUCg & -14.6 & 0.71 & $-96.1,67.0$ & 8.7 & 0.26 & $-7.0,24.5$ & -2.4 & 0.74 & $-17.5,12.6$ \\
\hline & CAR & 0.64 & 0.93 & $-14.7,16.0$ & -1.9 & 0.09 & $-4.1,0.3$ & -1.9 & 0.09 & $-4.2,0.3$ \\
\hline & Delta & 2.1 & 0.76 & $-12.0,17.0$ & -0.7 & 0.67 & $-4.2,2.7$ & -1.9 & 0.20 & $-5.0,1.1$ \\
\hline \multirow[t]{4}{*}{ Appetite increased } & $\mathrm{HCC}$ & -4.3 & 0.28 & $-12.2,3.7$ & 0.4 & 0.67 & $-1.4,2.2$ & 0.9 & 0.25 & $-0.7,2.4$ \\
\hline & AUCg & 28.4 & 0.48 & $-52.6,109.5$ & 0.7 & 0.94 & $-15.7,17.0$ & 6.4 & 0.27 & $-5.4,18.3$ \\
\hline & CAR & 0.4 & 0.96 & $-14.9,15.7$ & 0.3 & 0.80 & $-2.1,2.7$ & 1.4 & 0.22 & $-0.7,3.0$ \\
\hline & Delta & 0.1 & 0.99 & $-14.1,14.2$ & 0.6 & 0.74 & $-2.9,4.1$ & 1.5 & 0.23 & $-1.0,3.9$ \\
\hline \multirow[t]{4}{*}{ Fatigue/Energy level } & $\mathrm{HCC}$ & -1.3 & 0.65 & $-7.1,4.5$ & -0.4 & 0.63 & $-2.3,1.4$ & -3.0 & 0.11 & $-6.9,0.8$ \\
\hline & AUCg & 28.4 & 0.48 & $-52.6,109.5$ & 1.7 & 0.87 & $-20.5,24.0$ & 21.6 & 0.07 & $-2.5,45.9$ \\
\hline & CAR & 0.4 & 0.96 & $-15.0,15.7$ & 1.2 & 0.44 & $-1.9,4.4$ & -0.6 & 0.77 & $-4.8,3.6$ \\
\hline & Delta & 0.1 & 0.99 & $-14.1,14.2$ & 4.3 & $0.03^{*}$ & $0.3,8.3$ & -1.1 & 0.69 & $-6.7,4.5$ \\
\hline \multirow[t]{4}{*}{ Memory Impairment } & $\mathrm{HCC}$ & 1.7 & 0.66 & $-6.3,9.8$ & -0.4 & 0.54 & $-1.9,1.0$ & 2.4 & 0.08 & $-0.3,5.2$ \\
\hline & AUCg & $0.0 \S$ & - & - & 2.2 & 0.76 & $-12.8,17.3$ & 6.7 & 0.51 & $-14.3,27.7$ \\
\hline & CAR & $0.0 \S$ & - & - & -0.5 & 0.62 & $-2.7,1.6$ & 0.4 & 0.80 & $-3.0,3.81$ \\
\hline & Delta & $0.0 \S$ & - & - & 0.9 & 0.53 & $-2.0,3.9$ & 0.3 & 0.90 & $-4.2,4.7$ \\
\hline \multirow[t]{4}{*}{ Weight decreased } & HCC & 0.7 & 0.80 & $-5.1,6.5$ & -1.1 & $0.05^{*}$ & $-2.1,0.0$ & 0.1 & 0.92 & $-1.8,2.0$ \\
\hline & AUCg & -15.1 & 0.60 & $-73.5,43.3$ & 0.4 & 0.94 & $-10.2,10.9$ & 2.8 & 0.66 & $-10.5,16.1$ \\
\hline & CAR & -3.5 & 0.51 & $-14.5,7.5$ & -0.8 & 0.28 & $-2.3,0.7$ & -0.7 & 0.48 & $-2.7,1.3$ \\
\hline & Delta & $0.0 \S$ & - & - & 0.1 & 0.95 & $-2.2,2.3$ & -0.4 & 0.75 & $-3.1,2.3$ \\
\hline \multirow[t]{4}{*}{ Weight increased } & $\mathrm{HCC}$ & $0.0 \S$ & - & - & -0.2 & 0.81 & $-1.6,1.3$ & -0.3 & 0.71 & $-1.8,1.3$ \\
\hline & AUCg & $0.0 \S$ & - & - & -2.3 & 0.72 & $-15.7,11.1$ & -0.1 & 0.98 & $-10.7,10.5$ \\
\hline & CAR & $0.0 \S$ & - & - & 1.3 & 0.17 & $-0.6,3.2$ & 1.2 & 0.13 & $-0.4,2.8$ \\
\hline & Delta & $0.0 \S$ & - & - & 1.5 & 0.30 & $-1.4,4.3$ & 1.6 & 0.12 & $-0.5,3.7$ \\
\hline \multirow[t]{4}{*}{ Major life events } & HCC & 0.0 & 0.63 & $0.0,0.1$ & 0.0 & 0.43 & $0.0,0.1$ & 0.0 & 0.44 & $0.0,0.0$ \\
\hline & AUCg & 0.0 & 0.82 & $-0.1,0.1$ & 0.0 & 0.40 & $0.0,0,1$ & -0.1 & 0.14 & $-0.1,0.0$ \\
\hline & CAR & 0.0 & 0.51 & $0.0,0.1$ & 0.0 & 0.33 & $-0.1,0.0$ & 0.0 & 0.89 & $0.0,0.0$ \\
\hline & Delta & 0.0 & 0.74 & $0.0,0.1$ & 0.0 & 0.19 & $-0.1,0.0$ & 0.0 & 0.76 & $0.0,0.0$ \\
\hline \multirow[t]{4}{*}{ Daily hassles ${ }^{* *}$} & $\mathrm{HCC}$ & 0.0 & 0.96 & $-0.2,0.1$ & 0.0 & 0.79 & $-0.1,0.1$ & 0.0 & 0.56 & $-0.1,0.1$ \\
\hline & AUCg & -0.4 & 0.51 & $-1.9,1.0$ & -0.5 & 0.29 & $-1.5,0.5$ & 0.2 & 0.30 & $-0.2,0.7$ \\
\hline & CAR & -0.1 & 0.53 & $-0.3,0.2$ & 0.0 & 0.79 & $-0.2,0.1$ & 0.0 & 0.83 & $-0.1,0.1$ \\
\hline & Delta & 0.0 & 0.98 & $-0.3,0.3$ & -0.2 & $0.05^{*}$ & $-0.3,0.0$ & 0.0 & 0.94 & $-0.2,0.1$ \\
\hline
\end{tabular}

cortisol concentration alterations in A-MDE memory impairment with increased long-term cortisol levels, fatigue with decreased long-term but increased short-term cortisol - these were not strong associations and were not statistically significant. On the other hand, mid-nocturnal insomnia was significantly associated with the main observed cortisol alteration, namely decreased short-term cortisol output (AUCg) in A-MDE.
Other clinical features, such as hypersomnia, daily hassles, diurnal mood variation and weight decrease, were associated with cortisol concentration alterations in NA-MDE. Finally, amongst all of those overlapping and non-overlapping symptoms that were associated with cortisol level variations in this study, only increased fatigue and mid-nocturnal insomnia were also related to an increased number of daily hassles in A-MDE. 


\begin{tabular}{|c|c|c|c|c|c|c|c|c|c|c|c|}
\hline \multirow{2}{*}{\multicolumn{3}{|c|}{ Variables }} & \multicolumn{9}{|c|}{ Groups } \\
\hline & & & \multicolumn{3}{|c|}{ Controls } & \multicolumn{3}{|c|}{ Non-atypical MDE } & \multicolumn{3}{|c|}{ Atypical MDE } \\
\hline Clinical variables $\dagger$ & \multicolumn{2}{|c|}{ Cortisol measureł } & $\beta$ & $P$-value & Cl95\% & $\beta$ & $P$-value & $\mathrm{Cl} 95 \%$ & $\beta$ & $P$-value & $\mathrm{Cl} 95 \%$ \\
\hline \multirow[t]{8}{*}{ Diurnal reactivity } & \multirow[t]{2}{*}{$\mathrm{HCC}$} & Worse in AM & $0.0 \S$ & - & - & -2.6 & 0.13 & $-5.9,0.8$ & -1.9 & 0.50 & $-7.8,4.0$ \\
\hline & & Worse in PM & $0.0 \S$ & - & - & -3.7 & $0.03^{*}$ & $-7.1,-0.3$ & -1.6 & 0.46 & $-6.3,3.0$ \\
\hline & \multirow[t]{2}{*}{$\mathrm{AUC}_{\mathrm{g}}$} & Worse in AM & $0.0 \S$ & - & - & -15.4 & 0.34 & $-48.3,17.4$ & -14.7 & 0.46 & $-56.7,27.2$ \\
\hline & & Worse in PM & $0.0 \S$ & - & - & -25.6 & 0.16 & $-62.6,11.4$ & 1.8 & 0.90 & $-31.1,34.9$ \\
\hline & \multirow[t]{2}{*}{ CAR } & Worse in AM & $0.0 \S$ & - & - & -2.7 & 0.25 & $-7.5,2.1$ & -1.3 & 0.65 & $-7.6,4.9$ \\
\hline & & Worse in PM & $0.0 \S$ & - & - & -0.4 & 0.88 & $-5.8,5.0$ & 1.6 & 0.53 & $-3.7,6.9$ \\
\hline & \multirow[t]{2}{*}{ Delta } & Worse in AM & $0.0 \S$ & - & - & -0.1 & 0.98 & $-7.0,6.8$ & 1.2 & 0.77 & $-7.2,9.5$ \\
\hline & & Worse in PM & $0.0 \S$ & - & - & 1.7 & 0.66 & $-6.1,9.5$ & 2.8 & 0.40 & $-4.1,9.9$ \\
\hline
\end{tabular}

HCC: hair cortisol concentration; CAR: Cortisol awakening response over 60 min measured as area under the curve with respect to increase (AUCi); DELTA-Cortisol: increase in cortisol over baseline at $30 \mathrm{~min}$ after awakening; AUCg: total daily cortisol output measured as area under the curve with respect to ground.

$\dagger$ Scores were obtained using QIDS-C16 scale, apart from diurnal reactivity which was obtained using HAMD-21 scale.

$¥ A \cup C g$, CAR and DELTA are expressed in nm $h$ and HCC is expressed in $\mathrm{pg} / \mathrm{mg}$.

§Omitted because of collinearity. Cl95\%: 95\% Confidence interval.

qScores were obtained using RLCQ scale.

${ }^{* *}$ Scores were obtained using the hassles scale.

${ }^{*} P$ significant at 0.05 level.

We did not find elevated short-term or long-term cortisol secretion, nor short-term cortisol reactivity, in non-atypical depression, which was contrary to our hypothesis. This may be due to several factors. Criteria for non-atypical depression are less well defined than atypical clinical features, and indeed, there is no operationalised definition of 'typical' depression per se. The non-atypical group will be somewhat more heterogeneous and comprise patients with a number of subtypes of depression, including melancholic, psychotic, anxiousdistress, peripartum onset and seasonal pattern as well as those with non-specific features. Therefore, the presence of several subtypes of major depression likely contributed to greater heterogeneity in the composition of this non-atypical group. It is likely that focusing more specifically on the specific non-atypical subtypes that have been previously associated with high levels of cortisol, such as melancholic (44) or psychotic depression (45) would increase concordance with previous results. It is also important to highlight that ADDS criteria for non-atypical depression included participants with simple mood-reactive depression without any other factors sufficient to diagnose atypical depression. Thus, around one-quarter of the patients within the non-atypical group had preserved mood reactivity, one of the main criteria for the diagnosis of atypical depression, further increasing the heterogeneity within this group. It is also possible that increased cortisol levels are not as frequent a finding in studies of patients with depression recruited from community and outpatient settings rather than in-patient settings. Finally, the use of hair specimens to measure chronic cortisol concentrations is a relatively new tool, and there remain uncertainties regarding the effects of many potential confounding factors (15, 46). As a result, other biological sources, such as fingernail specimens, might ultimately be better suited to measure chronic cortisol levels in affective disorders $(47,48)$.

It is also possible that methodological issues surrounding the use of hair samples may have affected our results. Whilst cortisol concentrations in hair are not significantly related to gender, hair colour and age group $(49,50)$, other covariates may affect its values. People of different ethnicities, for instance, have different rates of hair growth (51), whereas it is standard practice to estimate growth rates as $1 \mathrm{~cm} /$ month (52). Furthermore, there are still some concerns as to whether hair cortisol provides a valid index of long-term systemic cortisol. Although the majority of the studies strongly support that assumption $(12,53-55)$, some others have also suggested that local cortisol production may be an additional source of hair cortisol over and above systemic sources (56). Another factor is whether the secretion of sweat in the scalp affects hair cortisol measurements. Nevertheless, Grass et al. (57) demonstrated that this effect can be minimised by measuring cortisol from the hair shaft where it is least likely to be altered. Thus, in the current study, we did indeed extract cortisol from the hair shaft, and we removed contamination from sources of cortisol like sebaceous and sweat glands by washing hair samples with methanol before cortisol extraction.

The washout effect is another factor that could potentially affect the results of hair cortisol 
analyses, as cortisol levels in hair decrease over time (58). However, this effect is not readily apparent in the first $3 \mathrm{~cm}$ of hair closest to the root, which was the hair segment we measured in this study (58). Other factors such as the effect of natural hair colour seem to have no impact on the levels of cortisol in hair. On the other hand, the effect of cosmetic hair treatments is still controversial $(13,20,46)$.

The saliva results in the atypical group support previous studies in depressed participants with similar clinical features that have found lowered cortisol levels using the same AUCg measure of total daily salivary cortisol output (7). However, we have now extended these findings by also including $\mathrm{HCC}$ as a measure of long-term cortisol aggregated over a 3-month period, finding that atypical depression showed a more specific pattern of cortisol secretion, namely normal levels of cortisol in hair samples, and low levels of cortisol in saliva measured across the course of a day. There may be several explanations for this ostensibly divergent pattern of results. It could be that the lack of difference between atypical and non-atypical groups in hair cortisol reflects the lack of difference seen in cortisol reactivity measures, such as the CAR and DELTA: as circulating cortisol is much higher during these periods, for example being sixfold higher during the CAR than during the day, this may contribute more to the aggregated cortisol levels in hair and potentially blur other components of cortisol release that do differ. However, a recent study (59) demonstrated that HCC correlates most closely with salivary cortisol total output measures but does not correlate with salivary cortisol reactivity measures, such as the CAR. Thus, it appears that the evidence to date does not support the notion that HCC is mainly driven by these components of the HPA axis rhythm.

Another possible explanation for our dissimilar $\mathrm{HCC}$ and AUCg results in A-MDE may come from the conceptualisation that the 'reactivity' of this disorder may not be only from a phenomenological point of view (preserved mood reactivity) but also a neurobiological one (cortisol reactivity). It is possible to speculate that participants with this disorder possess low basal cortisol levels, but experience large spikes of cortisol release on some days, creating normal averaged levels when assessed over long time periods as with hair specimens. Future longitudinal studies would be necessary to investigate this possibility and confirm whether patients with atypical depression do indeed show these periods of neurobiological hyperreactivity. Of note was that we did not find cortisol hyperreactivity in the CAR or the DELTA in the atypical depression group.

If spikes of heightened cortisol release are present, based on this study's results, it may be possible to consider that the increased number of daily 'hassles' that participants with atypical features report could explain their cortisol hyperreactivity. In fact, previous studies have shown that not all depressed patients show a heightened cortisol response to psychosocial stress (60), but that this response could be more distinctive of patients with atypical depression. Indeed, this group of patients has shown a heightened neurobiological reactivity to events that emulate stressful situations, such as increased adrenocorticotropic hormone responses to the corticotropin-releasing factor challenge test (61). Thus, there is evidence of an underlying heightened sensitivity of the HPA axis in atypical depression, which in the presence of this observed increased frequency of daily 'hassles' could explain intermittent periods of increased cortisol levels. However, other causes and explanations need to be explored.

It is also important to highlight that in the present study, we found daily hassles were only associated with decreased short-term cortisol reactivity (DELTA) in NA-MDE, rather than being associated with any cortisol parameters in A-MDE (Table 4). Instead, it was an unexpected type of sleep disturbance, namely mid-nocturnal insomnia, that was specifically associated with decreased AUCg in A-MDE, rather than the definitional type of sleep disturbance in atypical depression (hypersomnia). By contrast, hypersomnia was related to cortisol reactivity (CAR) and short-term cortisol output (AUCg) variations in the heterogeneous NA-MDE group. It may be that in the presence of hypersomnia, aspects of sleep disturbance like mid-nocturnal insomnia are overlooked, whereas our results suggest that this type of insomnia might be intimately linked to the cortisol level alteration (decreased AUCg) found in A-MDE. Although we cannot disentangle cause and effect in the current study, it is known that there are internal 'biological clocks' that control several body mechanisms in humans, such as body temperature, blood pressure, metabolism, sleep rhythms and the release of hormones, such as cortisol, which could provide an underlying mechanism linking these alterations (62). Scheer \& Buijs (63) have also shown that light has a strong impact on increasing morning cortisol peak. Furthermore, Yehuda et al. (64) have discussed how chronobiological alterations in depression may reflect dysregulation of the hypothalamic-pituitary-adrenal (HPA) axis. 
As a possible parallel of this potential cortisol rhythm alteration in A-MDE, several studies have reported groups of patients that present with a neurobiological condition described as transient generalised glucocorticoid hypersensitivity, which is characterised by clinical manifestations of Cushing syndrome such as hypertension and diabetes but with low levels of cortisol in blood or saliva (65-67). These participants showed increased tissue sensitivity to glucocorticoids and compensatory hypoactivation of the hypothalamic pituitary adrenal axis (67). Other research has suggested that participants with atypical depression have hyposecreation of corticotropin-releasing factor (68). According to these authors, the low cortisol levels that A-MDE participants may present is secondary to decreased levels of corticotropinreleasing factor. For others, however, low cortisol levels in depression are thought to result from desensitisation of corticotropin-releasing factor receptors in the hypothalamus due to increased cortisol production at the beginning of the affective disease (69). Although there have been to date no longitudinal data to address this question, decreased long-term cortisol levels have been associated with childhood trauma, irrespective of current depressive episode (19). A potential desensitisation of corticotropin-releasing factor receptors in A-MDE may then be explained by an increased cortisol production at the beginning of the stress response, which could be caused by early life trauma; this is, in turn, another environmental factor highly associated with MDE (Table 1).

Nevertheless, neither recent life events nor early life trauma were clinical features that significantly varied between the two groups of depression we studied. On the other hand, other environmental factors did: our results showed a significant association between atypical depression and the number, but not severity of 'hassles'. According to the authors of the Hassles Scale, this result suggests that participants with atypical features are under a significantly higher risk of developing stress-related bodily disorders than the non-atypical group.

We have recently suggested that stressful and non-stressful environmental factors can be distinguished on the basis of effects on long-term cortisol output results using hair specimens (15). Environmental factors that are able to trigger significant long-term increases (normal stress response) or decreases (abnormal stress response) of cortisol are those which may qualify as true 'stressors'. The present study did not find an increased number of daily hassles to be associated with long-term cortisol concentration alterations in any group, arguing against the likelihood that daily hassles are the predominant factor underlying the observed neurobiological changes.

The significant association between A-MDE and those symptoms (concentration impairment and fatigability) that overlap between MDE and CFS strengthens the link between this disorder and somatic symptom disorders such as chronic fatigue syndrome and fibromyalgia in which such complaints are central. Moreover, several somatic symptom disorders have been associated with lowered cortisol output (70-74) and CAR (75). There were, however, conflicting results regarding the inter-relationships between these symptoms, daily hassles and cortisol level alterations in AMDE. Daily hassles were associated with fatigue, although not with impaired concentration, suggesting that even though these environmental factors do not seem to explain the cortisol level variations found in A-MDE (decreased AUCg), they might be linked to the presence of one of its commonly associated symptoms, fatigue. In turn, higher numbers of daily hassles were positively correlated with mid-nocturnal insomnia, which we have already discussed was a key factor linked to the observed reduced short-term cortisol levels in A-MDE. The nature of the correlations between fatigue and cortisol parameters did differ between short- and long-term cortisol measures, but were at trend level only and we would caution against making any firm interpretations of these links without further confirmatory data. Similarly, the trend for the other overlapping symptom (impaired memory) to be associated with an increased long-term measure of cortisol (HCC) does fit with the suggestions of a potential role of hypercortisolism in the neurocognitive impairment seen in some affective disorders (76), but should also be treated with caution at this stage.

Further overlap between A-MDE and somatic symptom disorders derives from the already mentioned observation of a higher prevalence in the number of common day-to-day environmental disturbances, which is a risk factor for developing a stress-related bodily disorder, a category to which, for some authors, chronic fatigue syndrome and fibromyalgia belong (25). Finally, rates of depressive illness are high in those with somatic symptom disorders; for example, up to half of patients with chronic fatigue syndrome have a diagnosable depressive illness using standard diagnostic criteria, even excluding overlapping clinical features (77). All the aforementioned neurobiological and clinical data strengthen the idea that patients with atypical depression and patients with somatic 


\section{Herane-Vives et al.}

symptoms disorders show considerable overlapping features (27).

\section{Limitations}

We should acknowledge some limitations of this study. In terms of saliva sampling, we measured salivary cortisol on 1 day only, whereas more recent guidelines suggest that 2 days of sampling are preferable (78). In addition, the saliva samples were taken 1 week after hair samples, and we cannot exclude that other factors may have had some effect during this period between taking the two types of samples. We are also limited in so far as our measures of stress, and life events are only as good as self-reported measures allow in this context. Whilst the Hassles scale and the RLCQ are widely used and validated, they are not as definitive as other interview- and contextual-based measures such as the Life Events and Difficulties Schedule (79). Similarly, fatigue and memory/concentration symptoms were measured using single items which, although part of a validated scale, the QIDS-C, may not fully capture the full nature and extent of these symptom categories. Nevertheless, it has been demonstrated that using a single fatigability item can be a reliable tool in the assessment of fatigue (80).

Whilst the sample size of 71 depressed patients is moderately sized, it is a strength that we have been able to exclude psychotropic medication effects on the results. To do this, we were not able to impose a strict symptom severity criterion (e.g. on the HDRS17) as it is rarely possible to find more severely ill but untreated patients among an out-patient population. Furthermore, we should also note that whilst participants taking psychotropic medication, including benzodiazepines and hypnotics, and any kind of steroid were excluded from this sample, they were still included if they had taken other kinds of medication. None of these medications were likely to have an effect on the HPA; the list included paracetamol, antihistamines, analgesics, levothyroxine, omeprazole, vitamins and other supplements, such as calcium, and only a minority of subjects was taking any medication. The range of medicines used was broad; hence, the groups were too small to undertake any form of statistical analysis. Nevertheless, although a large confounding effect of these other types of medication on cortisol results is unlikely, a confounding effect cannot be completely excluded. On the other hand, excluding participants on any type of medication would have risked introducing bias to the results by selecting a superhealthy population (81).

\section{Implications and summary}

Contrary to our expectation, increased cortisol levels were not found to be a chronic phenomenon in major depression, at least using hair specimens. Future studies should aim to specifically investigate chronic cortisol secretion patterns in specific non-atypical subtypes of depression such as psychotic and melancholic depression using hair or alternative specimens, for example fingernails. The atypical depression group was found to share several clinical and neurobiological features with somatic symptom disorders. Future investigations should also directly compare atypical depression with chronic fatigue syndrome using chronic cortisol measures. It may also be of interest to investigate whether the specific pattern of acute and chronic cortisol levels is also shared between these two illnesses, and also to determine the possible effects of treatment on these parameters. Previous studies have shown an association between atypical features and poor outcome following antidepressant treatment (5). If links between cortisol status and outcome are shown, this might have therapeutic implications, as has been shown in chronic fatigue syndrome $(82,83)$. From a clinical perspective, it appears important to focus on reducing environmental factors as a way to aid clinical improvement. Our results also suggest that future studies should pay more attention to an overlooked type of insomnia - mid-nocturnal insomnia - and its potential link with cortisol level alterations. It may be that this type of sleep disturbance, rather than hypersomnia, is more closely associated with the observed cortisol level alteration found in A-MDE.

Future studies may also improve understanding by comparing cortisol levels between different depressive item scores, such as fatigue and memory impairment, rather than diagnostic constructs such as atypical depression that we have used, in addition to life events or daily hassles, in order to pinpoint possible reasons for low and high cortisol levels. Another option would be to turn things around and start by defining groups on the basis of cortisol parameters, and then regress a series of dependent variables, such as symptom profiles and the presence and/or effects of different types of environmental factors, on cortisol measures. Finally, there may be effects of sex that affect results, as is the case with somatic symptom disorders (74). Thus, we also ran an exploratory and preliminary analysis about gender differences in terms of short- and long-term cortisol levels in A-MDE and NA-MDE, which suggested that the AUCg differences were mainly driven by large cortisol reductions in the group of 
males with A-MDE. However, their reduced number and the post hoc nature of this analysis did not allow us to draw any significant conclusion. Therefore, future studies may investigate the role of gender in atypical depression.

To summarise, the results in this article contribute to a fuller understanding of clinical and neurobiological features of atypical and non-atypical major depression. The negative findings in the non-atypical group weaken the possibility of considering increased cortisol levels a general biomarker in depression, at least for out-patients and using hair samples. In contrast, a specific pattern of cortisol secretion could be defined in the atypical depression group with normocortisolism in hair and decreased cortisol levels in saliva. This combination of lowered basal cortisol levels in shortterm measures and normal levels in longer term averaged measures might be explained by a hypothesised pattern of neurobiological hyperreactivity, or by mid-nocturnal insomnia, which in turn was also closely related to the increased number of daily hassles that these patients exhibited.

\section{Acknowledgements}

This research was funded by departmental funds generated by AJC, AHV and AHY and start up funds from the Academy of Medical Sciences to DA (Ref. AMS-SGCL8). AHV was supported by a Chilean Bicentennial Fund Scholarship from the Bicentennial Fund for Human Capital Development (Becas Chile) and by the Psychiatric Research Trust. AJC, TW and AHY are supported by the NIHR Biomedical Research Centre at South London and Maudsley NHS Foundation Trust and King's College London. This study represents independent research part funded by the NIHR/Wellcome Trust, King's Clinical Research Facility and the NIHR Biomedical Research Centre at South London and Maudsley NHS Foundation Trust and King's College London. The views expressed are those of the author(s) and not necessarily those of the NHS. The authors would like to thank the staff of the NIHR/Wellcome Trust Clinical Research Facility at King's College Hospital and the Clínica Psiquíatrica Universitaria and Oficina de Apoyo a la Investigacion Clínica (OAIC) of University of Chile for their support in the conduct of the study. We are grateful to Mrs Irene Papadopoulos for performing the saliva cortisol Immunoassays and Miss Carolina Afandi for her technical assistance. We thank the Mental Health Research Network for contributing to this study and all participants in this study for their support. The funders had no role in the design and conduct of the study; collection, management, analysis and interpretation of the data; preparation, review or approval of the manuscript; and decision to submit the manuscript for publication.

\section{Declaration of interest}

AJC has in the last 3 years received honoraria for speaking from Astra Zeneca (AZ) and Lundbeck, honoraria for consulting from Janssen, Allergan and Livanova and research grant support from Lundbeck. AHY has given paid lectures and sits on advisory boards for all major pharmaceutical companies with drugs used in affective and related disorders. DA has received travel grants from Janssen-Cilag and Servier. No other disclosures were reported.

\section{References}

1. Knorr U, Vinberg M, Kessing LV, Wetterslev J. Salivary cortisol in depressed patients versus control persons: a systematic review and meta-analysis. Psychoneuroendocrinology 2010;35:1275-1286.

2. Pariante CM. Risk factors for development of depression and psychosis: glucocorticoid receptors and pituitary implications for treatment with antidepressant and glucocorticoids. Ann N Y Acad Sci 2009;1179:144-152.

3. Schatzberg A, Garlow S, Nemeroff C. Molecular and cellular mechanisms in depression. The Fifth Generation of Progress. 2002. Retrieved from http://www.acnp.org/Doc s/G5/CH72_1039-1050.pdf

4. Sullivan P. Latent class analysis of lifetime depressive symptoms in the national comorbidity survey. Am J Psychiatry 1998;155:1398-1406.

5. Parker G, Roy K, Mitchell P. Atypical depression: a reappraisal. Am J Psychiatry 2014. Retrieved from http:/ajp. psychiatryonline.org/doi/10.1176/appi.ajp.159.9.1470

6. American Psychiatric Association. DSM 5. American Psychiatric Association. 2013. Retrieved from https://books. google.com/books?id =_VzzAgAAQBAJ\&pgis $=1$

7. Lamers $\mathrm{F}$, Vogelzangs $\mathrm{N}$, Merikangas KR, de Jonge $\mathrm{P}$, Beekman ATF, Penninx BWJH. Evidence for a differential role of HPA-axis function, inflammation and metabolic syndrome in melancholic versus atypical depression. Mol Psychiatry 2012;18:692-699.

8. Gold P, Chrousos G. The endocrinology of melancholic and atypical depression: relation to neurocircuitry and somatic consequences. Proc Assoc Am Physicians 1999; 111:22-34.

9. Vreeburg S, Hoogendijk WJ, van Pelt J et al. Major depressive disorder and hypothalamic-pituitary-adrenal axis activity: results from a large cohort study. Arch Gen Psychiatry 2009;66:617-626.

10. O'Keane V, Frodl T, Dinan TG. A review of Atypical depression in relation to the course of depression and changes in HPA axis organization. Psychoneuroendocrinology 2012;37:1589-1599.

11. Russell E, Koren G, Rieder M, Van Uum S. Hair cortisol as a biological marker of chronic stress: current status, future directions and unanswered questions. Psychoneuroendocrinology 2012;37:589-601.

12. D'Anna-Hernandez KL, Ross RG, Natvig CL, LauDENSLAGER ML. Hair cortisol levels as a retrospective marker of hypothalamic-pituitary axis activity throughout pregnancy: comparison to salivary cortisol. Physiol Behav 2011;104:348-353.

13. Sauvé B, Koren G, Walsh G, Tokmakejian S, Van Uum SHM. Measurement of cortisol in human hair as a biomarker of systemic exposure. Clin Investig Med, 2007;30: E183-E191.

14. XIE Q, GaO W, Li $\mathbf{J}$ et al. Correlation of cortisol in 1-cm hair segment with salivary cortisol in human: hair cortisol as an endogenous biomarker. Clin Chem Lab Med CCLM FESCC 2011;49:2013-2019.

15. Herane Vives A, De Angel V, Papadopoulos A et al. The relationship between cortisol, stress and psychiatric illness: new insights using hair analysis. J Psychiatr Res, 2015;70:38-49. 


\section{Herane-Vives et al.}

16. Stalder T, Kirschbaum C. Analysis of cortisol in hair-state of the art and future directions. Brain Behav Immun 2012;26:1019-1029.

17. Dettenborn L, Muhtz C, Skoluda $\mathrm{N}$ et al. Introducing a novel method to assess cumulative steroid concentrations: increased hair cortisol concentrations over 6 months in medicated patients with depression. Stress, 2012;15:348353.

18. Wei J, Sun G, Zhao L et al. Analysis of hair cortisol level in first-episodic and recurrent female patients with depression compared to healthy controls. J Affect Disord 2015; 175:299-302.

19. Hinkelmann K, Muhtz C, Dettenborn L et al. Association between childhood trauma and low hair cortisol in depressed patients and healthy control subjects. Biol Psychiatry, 2013;74:e15-e17.

20. Manenschijn L, Spijker A, Koper J. Long-term cortisol in bipolar disorder: associations with age of onset and psychiatric co-morbidity. Psychoneuroendocrinology, 2012;37:1960-1968.

21. Pochigaeva K, Druzhrova T, Yakovlev A et al. Hair cortisol as a marker of hypothalamic-pituitary-adrenal Axis activity in female patients with major depressive disorder. Metabolic Brain 2017;32:577-583.

22. Griep EN, Boersma JW, Lentjes EG, Prins AP, Van der Korst JK, De Kloet ER. Function of the hypothalamicpituitary-adrenal axis in patients with fibromyalgia and low back pain. J Rheumatol 1998;25:1374-1381.

23. Papadopoulos AS, Cleare AJ. Hypothalamic-pituitaryadrenal axis dysfunction in chronic fatigue syndrome. Nat Rev Endocrinol 2012;8:22-32.

24. Roberts AD, Wessely S, Chalder T, Papadopoulos A, Cleare AJ. Salivary cortisol response to awakening in chronic fatigue syndrome. Br J Psychiatry 2004;184:136141

25. Fries E, Hesse J, Hellhammer J, Hellhammer DH. A new view on hypocortisolism. Psychoneuroendocrinology 2005;30:1010-1016.

26. Gold PW, Chrousos GP. Organization of the stress system and its dysregulation in melancholic and atypical depression: high vs low $\mathrm{CRH} / \mathrm{NE}$ states. Mol Psychiatry 2002; 7:254-275.

27. Juruena MF, Cleare AJ. Overlap between atypical depression, seasonal affective disorder and chronic fatigue syndrome Superposição entre depressão atípica, doença afetiva sazonal e síndrome da fadiga crônica. Rev Bras Psiquiatr 2007;44:19-26.

28. Heim C, Ehlert U, Hellhammer DH. The potential role of hypocortisolism in the pathophysiology of stress-related bodily disorders. Psychoneuroendocrinology 2000;25: $1-35$.

29. Wise T, Arnone D, Marwood L, Zahn R, Lythe KE, Young AH. Recruiting for research studies using online public advertisements: examples from research in affective disorders. Neuropsychiatr Dis Treat 2016;12:279-285.

30. Sheehan DV, Lecrubier Y, Sheehan KH et al. The MiniInternational Neuropsychiatric Interview (M.I.N.I.): the development and validation of a structured diagnostic psychiatric interview for DSM-IV and ICD-10. J Clin Psychiatry, 1998;59(Suppl 2):22-33.

31. Hamilton M. A rating scale for depression. J Neurol Neurosurg Psychiatry 1960;23:56.

32. Rush AJ, Trivedi MH, Ibrahim HM et al. The 16-Item Quick Inventory of Depressive Symptomatology (QIDS), clinician rating (QIDS-C), and self-report (QIDS-SR): a psychometric evaluation in patients with chronic major depression. Biol Psychiatry 2003;54:573-583.
33. Young R, Biggs J, Ziegler V, Meyer D. A rating scale for mania: reliability, validity and sensitivity. Br J Psychiatry 1978; 133:429-435.

34. Stewart JW, McGrath PJ, Rabkin JG, Quitkin FM. Atypical depression: a valid clinical entity? Psychiatr Clin North Am, 1993;16:479-496.

35. Cusin C, YAng H, Yeung A, Fava M. Rating scales for depression. In: BAER L, BLAIS MA, eds. Handbook of clinical rating scales and assessment in psychiatry and mental health. Current clinical psychiatry. Totowa, NJ: Humana Press, 2010: 7-35.

36. Kanner A, Coyne J, Schaefer C, Lazarus RS. Comparison of two modes of stress measurement: daily hassles and uplifts versus major life events. J Behav Med 1981;4:1-39.

37. Miller M, Rahe R. Life changes scaling for the 1990s. J Psychosom Res 1997;43:279-292.

38. Bernstein D, Ahluvalia T, Pogge D, Handelsman L. Validity of the Childhood Trauma Questionnaire in an adolescent psychiatric population. J Am Acad Child Adolesc Psychiatry 1997;36:340-348.

39. Albermann M, Musshoff F, Aengenheister L, Madea B. Investigations on the influence of different grinding procedures on measured ethyl glucuronide concentrations in hair determined with an optimized and validated LC-MS/ MS method. Anal Bioanal Chem 2012;403:769-776.

40. Mondelli V, Dazzan P, Hepgul $N$ et al. Abnormal cortisol levels during the day and cortisol awakening response in first-episode psychosis: the role of stress and of antipsychotic treatment. Schizophr Res 2010;116:234-242.

41. Clow A, Thorn L, Evans P, Hucklebridge F. The awakening cortisol response: methodological issues and significance. Stress 2004;7:29-37.

42. Kunz-Ebrecht S, Kirschbaum C, Marmot M, Steptoe A. Differences in cortisol awakening response on work days and weekends in women and men from the Whitehall II cohort. Psychoneuroendocrinology 2004;29:516-528.

43. CoHen J. Statistical power analysis for the behavioral sciences. 2013. Retrieved from https://books.google.co. $\mathrm{uk} /$ books?hl $=$ en\&lr $=\& \mathrm{id}=$ rEe0BQAAQBAJ\&oi $=$ fnd $\& p g=\mathrm{PP} 1 \& d q=$ cohen + statistical + power + analysis $+\mathrm{f}$ or + the + behaviou\&ots $=$ suYSHwSQp6\&sig $=$ TU9Xrwl366j0gXip0Apf-FLaaf0

44. Carroll B, Cassidy F, Naftolowitz D et al. Pathophysiology of hypercortisolism in depression. Acta Psychiatr 2007;115:90-103.

45. Bhagwagar Z, Hafizi S, Cowen PJ. Increase in concentration of waking salivary cortisol in recovered patients with depression. Am J Psychiatry 2003;160:1890-1891.

46. Fischer S, Duncko R, Papadopoulos A, Hatch SL, Hotopf M, Cleare AJ. Sociodemographic, lifestyle, and psychosocial determinants of hair cortisol-Evidence from a south London community sample. Psychoneuroendocrinology 2016;71:74.

47. Herane-Vives A, Cleare AJ, Chang C-K et al. Cortisol levels in fingernails, neurocognitive performance and clinical variables in euthymic bipolar I disorder. World J Biol Psychiatry 2017;2017:1-12.

48. Izawa S, Miki K, Tsuchiya M et al. Cortisol level measurements in fingernails as a retrospective index of hormone production. Psychoneuroendocrinology 2015;54:24-30.

49. GAO W, XIE Q, JIN J et al. HPLC-FLU detection of cortisol distribution in human hair. Clin Biochem, 2010;43:677-682.

50. Raul J, Cirimele V, Ludes B, Kintz P. Detection of physiological concentrations of cortisol and cortisone in human hair. Clin Biochem 2004;37:1105-1111.

51. Loussounarn G, El Rawadi C, Genain G. Diversity of hair growth profiles. Int J Dermatol 2005;44(Suppl 1):6-9. 
52. Luo H, Hu X, Liu X et al. Hair cortisol level as a biomarker for altered hypothalamic-pituitary-adrenal activity in female adolescents with posttraumatic stress disorder after the 2008 Wenchuan earthquake. Biol Psychiatry, 2012;72:65-69.

53. Kalra S, Einarson A, Karaskov T, Van Uum S, Koren G. The relationship between stress and hair cortisol in healthy pregnant women. Clin Investig Med 2007;30:E103-E107.

54. van Holland BJ, Frings-Dresen MHW, Sluiter JK. Measuring short-term and long-term physiological stress effects by cortisol reactivity in saliva and hair. Int Arch Occup Environ Health 2012;85:849-852.

55. Van Uum ShM, Sauvé B, Fraser LA, Morley-Forster P, Paul TL, Koren G. Elevated content of cortisol in hair of patients with severe chronic pain: a novel biomarker for stress. Stress, 2008;11:483-488.

56. Sharpley C. Stress-linked cortisol concentrations in hair: what we know and what we need to know. Rev Neurosci 2012;23:111-121.

57. Grass J, Kirschbaum C, Miller R. Sweat-inducing physiological challenges do not result in acute changes in hair cortisol concentrations. Psychoneuroendocrinology, 2015;53:108-116.

58. Dettenborn L, Tietze A, Bruckner F, Kirschbaum C. Higher cortisol content in hair among long-term unemployed individuals compared to controls. Psychoneuroendocrinology, 2010;35:1404-1409.

59. Short S, Stalder T, Marceau K, Entringer S. Correspondence between hair cortisol concentrations and 30-day integrated daily salivary and weekly urinary cortisol measures. Psychoneuroendocrinology 2016;71:12-18.

60. Zorn JV, Schür RR, Boks MP, Kahn RS, Jö̈LS M, Vinkers $\mathrm{CH}$. Cortisol stress reactivity across psychiatric disorders: a systematic review and meta-analysis. Psychoneuroendocrinology 2017;77:25-36.

61. O'Keane V, Dinan T, Scott L, Corcoran C. Changes in hypothalamic-pituitary-adrenal axis measures after vagus nerve stimulation therapy in chronic depression. Biol Psychiatry 2005;58:963-968.

62. Price JL, Dembinska ME, Young MW, Rosbash M. Suppression of PERIOD protein abundance and circadian cycling by the Drosophila clock mutation timeless. EMBO J, 1995;14:4044-4049.

63. SCHEER FAJL, Buiss RM. Light affects morning salivary cortisol in humans. J Clin Endocrinol Metab 1999;84:3395-3398.

64. Yehuda R, Teicher M, Trestman R. Cortisol regulation in posttraumatic stress disorder and major depression: a chronobiological analysis. Biol Psychiatry 1996;40:79-88.

65. IIda S, Nakamura Y, FuJII $\mathrm{H}$ et al. A patient with hypocortisolism and cushing's syndrome-like manifestations: cortisol hyperreactive syndrome*. J Clin Endocrinol Metab 1990;70:729-737.

66. Krysiak R, Okopien B. Glucocorticoid hypersensitivity syndrome: a case report. West Indian Med J 2012;61:844-846.

67. Nicolaides NC, Lamprokostopoulou A, Polyzos A et al. Transient generalized glucocorticoid hypersensitivity. Eur J Clin Invest 2015;45:1306-1315.
68. Tsigos C, Chrousos G. Hypothalamic-pituitary-adrenal axis, neuroendocrine factors and stress. J Psychosom Res 2002;53:865-871.

69. BARDEN N. Implication of the hypothalamic-pituitary-adrenal axis in the physiopathology of depression. J Psychiatry Neurosci, 2004;29:185-193.

70. Buskila D, Press J. Neuroendocrine mechanisms in fibromyalgia-chronic fatigue. Best Pract Res Clin Rheumatol 2001;15:747-758.

71. Cleare AJ. The neuroendocrinology of chronic fatigue syndrome. Endocr Rev 2003;24:236-252.

72. Jerjes WK, Cleare AJ, Wessely S, Wood PJ, Taylor NF. Diurnal patterns of salivary cortisol and cortisone output in chronic fatigue syndrome. J Affect Disord 2005;87:299-304.

73. Roberts ADl, Papadopoulos AS, Wessely S, Chalder T, Cleare AJ. Salivary cortisol output before and after cognitive behavioural therapy for chronic fatigue syndrome. $\mathrm{J}$ Affect Disord 2009;115:280-286.

74. TaK LM, Cleare AJ, Ormel $J$ et al. Meta-analysis and meta-regression of hypothalamic-pituitary-adrenal axis activity in functional somatic disorders. Biol Psychol 2011;87:183-194.

75. Powell DJH, Liossi C, Moss-Morris R, Schlotz W. Unstimulated cortisol secretory activity in everyday life and its relationship with fatigue and chronic fatigue syndrome: a systematic review and subset meta-analysis. Psychoneuroendocrinology 2013;38:2405-2422.

76. Young A, Sahakian B, Robbins T, Cowen P. The effects of chronic administration of hydrocortisone on cognitive function in normal male volunteers. Psychopharmacology 1999; 145:260-266.

77. Parker A, Wessely S, Cleare A. The neuroendocrinology of chronic fatigue syndrome and fibromyalgia. Psychol Med 2001;31:1331-1345.

78. Stalder T, Kirschbaum C, Kudielka BM et al. Assessment of the cortisol awakening response: expert consensus guidelines. Psychoneuroendocrinology 2016;63:414-432.

79. Brown GW, George W, Harris T. Social origins of depression : a study of psychiatric disorder in women. Routledge, 2001. Retrieved from https://books.google.co.uk/books? $\mathrm{id}=$ pmAhsL44_60C\&printsec $=$ frontcover\&redir_esc $=$ $\mathrm{y} \# \mathrm{v}=$ onepage $\& \mathrm{q} \& \mathrm{f}=$ false

80. Kirsh K, Passik S, Holtsclaw E, Donaghy K, Theobald D. I get tired for no reason: a single item screening for cancerrelated fatigue. J Pain Symptom Manage 2001;22:931-937.

81. Сho K, Chung J, Cho S, Shin H, Jang I. Antihyperglycemic mechanism of metformin occurs via the AMPK/LXR [agr]/POMC pathway. Sci Rep 2015;5:8145.

82. Cleare A, Heap E, Malhi G, Wessely S, O'keane V, Miell J. Low-dose hydrocortisone in chronic fatigue syndrome: a randomised crossover trial. Lancet 1999;353:455-458.

83. Roberts A, Charler Ml, Papadopoulos A, Wessely S, Chalder T, Cleare AJ. Does hypocortisolism predict a poor response to cognitive behavioural therapy in chronic fatigue syndrome? Psychol Med 2010;40:515-522. 